\title{
SAND $80-0556$
}

$$
\text { Conf } 800448-1
$$

Gustavus J. Simmons

Sandia National Laboratories ${ }^{\ddagger}$ Albuquerque, New Mexico 87185

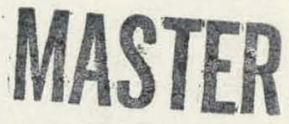

\section{Abstract}

The functions of secrecy, identification of the transmitter and of authentication of messages, and combinations of these, are in principle all achievable using either symmetric or asymmetric cryptosystems. Since secure communications depend on authenticated data exchanges between and/or authenticated actions by some of the parties to the communication, the fundamentals of authentication using symmetric or asymmetric techniques are developed. The surprising conclusion is that they differ only in the secure exchanges required to achieve further secure communications. The problem of authenticating a public-key directory is discussed in the light of these findings.

\section{Introduction}

It is now generally understood that an asymmetric (read also two-key or public-key) cryptosystem either makes it possible for a transmitter whose key has been compromised to still communicate with secrecy to a receiver whose key has been protected, or else for a receiver to authenticate a message so long as the transmitter's key has been kept secure. Obviously, in the first case authentication is impossible while in the second secrecy has been forfeited. Depending on the particular asymmetric encryption scheme used, either one or both of these capabilities may be achievable. The significance of asymmetric encryption to secure communications is based on these two capabilities, neither of which is possible in a symmetric (read one-key) cryptosystem.

On the other hand, the two most often claimed capabilities for asymmetric encryption are themselves impossible to realize (at least as usually described). In a symetric encryption system, the key must be exchanged between the transmitter and receiver prior to communications and in secret, since any compromise or exposure of the key precludes any form of secure communication. It is frequently claimed that asymmetric encryption has solved this key distribution problem by eliminating the need for a secure key distribution channel. This is not true - what has been accomplished is

+ This article sponsored by the U. S. Department of Energy under Contract DE-AC04-76DP00789.

* A U. S. Department of Energy Facility. that the keys exchanged need only be authenticated as opposed to being kept secret. Several investigators have proposed novel schemes for accomplishing this authentication function and a detailed discussion of the problems involved is given in this paper. It is also widely believed that asymmetric encryption has solved the problem of "signing" or "fingerprinting" digital messages through some variation of encrypting the message with the transmitter's secret key which results in a cipher that supposedly identifies the transmitter. Unlike a signature to a document however, whose authenticity cannot be affected by any subsequent act on the part of the signer, the authenticity of a digital signature can be made impossible to prove, by the transmitter either deliberately exposing his secret key or merely claiming that it has been compromised. Again, many investigators have devised protocols for specific applications that address this problem.1,2,3,13

Because of the two weaknesses just described, it is reasonable to say (of asymmetric encryption) that "there is less to it than meets the eye." This should not be interpreted as saying that there is nothing to it, since there are secure communications problems which cannot be solved using only symmetric encryption techniques, but which can be solved using either asymetric techniques or hybrid mixtures of the two. 4,5

This paper explores what can be accomplished toward solving some multiparty or network secure communications problems using both symmetric and asymmetric techniques. For example, the apparent paradox of being able, using asymmetric techniques, to establish secret communications with a party whose identity cannot be determined is well known. This problem is inherent in many network schemes such as in command and control systems. If the subordinate commander only needs to verify the authenticity (to his satisfaction) of a command from higher levels of command, there is no problem. The concern of the superior commander that his subordinate not be able to attribute a forged order to him can also be partially solved using either symmetric or asymmetric techniques. If, however, the subordinate commander needs the ability to later prove (to the satisfaction of a third party) that the command was given to him by his superior commander who may wish to disavow responsibility for the order, the problem is apparently insolvable.

Another example chosen to illustrate the intricacies of authentication is the problem of authenti- 


\section{DISCLAIMER}

This report was prepared as an account of work sponsored by an agency of the United States Government. Neither the United States Government nor any agency Thereof, nor any of their employees, makes any warranty, express or implied, or assumes any legal liability or responsibility for the accuracy, completeness, or usefulness of any information, apparatus, product, or process disclosed, or represents that its use would not infringe privately owned rights. Reference herein to any specific commercial product, process, or service by trade name, trademark, manufacturer, or otherwise does not necessarily constitute or imply its endorsement, recommendation, or favoring by the United States Government or any agency thereof. The views and opinions of authors expressed herein do not necessarily state or reflect those of the United States Government or any agency thereof. 


\section{DISCLAIMER}

Portions of this document may be illegible in electronic image products. Images are produced from the best available original document. 
cating a public-key directory - which is ironically the problem that public reys were supposed to avold. If the central authority who collects and publishes the public reys is trustworthy, several secure communications objectives for the subscribers can be achleved - but if he is deceitful, it appears impossible to authenticate the drectory!

The essential point developed here is that authentication is the basis for secure comountea$t$ ions and that if there are either trusted centers in the network which can vouch for the authenticity of some exchanges or $1 f$ some verfflably outhentic communications can be made then protocols can be devised to capitalize on this to realize many secure communications objectives. If, however, all parties in the network are regarded as potentially deceltful by the others, only very limited secure communications objectives can be realized. Unfortunately (for the systems designer), it is this latter case which describes most comercial, diploma$t i c$ and military commications networks.

\section{Secure Communications}

The confusion - and there is a great deal of 1t - about secure comunications is largely due to the fact that there are not only many different objectives for the transmitter and recelver either one of wham, or both, may on occasion wish to subvert the integrity of commins cations - but also on the part of the third parties. There is no "standard attacker or opponent" so that secure communications problems require the examination of many special cases. The approach which we shall use here will be to describe a small number of such problems and to explore means (sometimes called protocols) to realize thelr solution.

One of the simplest of secure communications situations is 1llustrated by the use of checks. There is a transmitter, T, i.e., the customer writing the check and a receiver, $R$, the bank. The third perties, $P$, are those intermediate persons who accept and forward the second-party (from their Fiewpoint) check or who may initiate forged checks against the customer's account. The bank is primarily concerned with the authenticity of a check that it accepts: that it was indeed issued by their custamer and for the amount and on the date and to the payee shown, i.e., that the check has not been altered. In this case it is usually assumed that if $P$ wishes to defeat the communication system between $T$ and $R$ at all, that bis objectives will be to either issue a fradulent check (forgery) or else to alter an authentic check which has come into his possession. The first is insured against by matching the signatures on the check against the outhenticated ones on file at the bank* and by the requirement for identification of the parties holding the check at each stage in the transaction.

This file is normally established in advance of the need for it by having the customer submit a copy of his signature at the time that the account is opened. Since signatures are legally considered to be unforgeable, it could be obtained in person at some later date should the customer question the
To protect against the second threat most banks now make available to their custcmers checks that indelibly indicate efforts at alteration such as erasure, scraping, lifting, etc., or the use of ink eradicators. This does indeed make it more difficult for $P$ to have an altered check accepted by the bank as authentic, but it makes it easy for him to cause an authentic check to be rejected. If the "tattle-tale" Indicates that an alteration has occurred, the bank would either refuse to accept the check and cause it to be returned, or else would more than likely accept it and delay the transfer of funds until the customer could be contacted to verify that the check was genuine, 1.e., to vouch for its authenticity. Although this 1sn't one of the secure communications problems that the safeguards in the checkeng system are designed to protect against, it isn't difficult to conceive of circumstances in which anyone of $T, P$ or $R$ might wish to cause an authentic check to be rejected and to incur the delay of special authentication. For example, my brokerage house credits my Liquid Assets Fund account with a deposit on the same day that they recelve my check and purchase order. I, as $T$, might wish to have the acceptance of the check delayed by the bank because then I could simultaneously receive interest on the principal at two institutions, which on a large deposit could be an incentive for delayling the verification of authenticity of my genuine check. In fact, I do receive such double interest during the float through no act of my own. On the other hand, on a very large withdrawal the bank might profit (at no risk to themselves) by delaying the transfer of funds because they could claim that they needed to verify the authenticity of the check which they clajed to show signs of tampering or on which they had caused the tattle-tale to show. Finally, if the check were being presented on a time demand payment, $P$ might profit by causing delay in the demand being satisfied. $P$ could be an agent of T's competition in a bid for a large contract for example where failure to pay on demand would automatically give the award to the next lower bidder, etc. There are even instances in which third parties bave maliciously interfered with check transactions by calling the bank and falsely identifying themselves os the custamer (?) and ordering a stop payment on a legitimate check or saying that a check (or checks) had been stolen or lost and should not be honored if presented. The (?) means that it may indeed have been the customer that called the bank, but that he later disavowed the call. The objectives in these examples by no means exhaust the possibilities. For example, an enterprising $P$ through whose hands T's checrs routinely pass could desensitize the authentication system by tripping the tattle-tale so frequently that $T$ and $R$ lose faith in it and raise their threshold for rejection to a point that $P^{\prime} s$ forgeries would be accepted. Even the possibility of intentional but illogical subversion of the system cannot be ruled out: there is a sizeable his-

Buthenticity of a charge against his account. The important point is that the compario on signature for the authentication must itself be authenticated, or be possible of authentication. 
tory of disgruntled employees committing acts to sabotage a system (often computing or data systems) in which no tanglble benefit accrues to the perpetrator.

Although the checiling system analogizes many of the security aspects of other secure communications problems, it is not an analog for privacy systems. One expects the bank to reep financial records confidential, but the checking system 1tself does not function to provide privacy. In most other respects though it is a paradigm for general secure communications systems in which the transmitter may wish to disavow genuine messages, or to 1ssue unauthentic messages which be will later authenticate as genulne, or attribute to third parties or to the receiver. Third parties may wish to utter forgeries or to alter genuine messages and. have them be accepted by the recelver as authentic, or to issue forgeries that are not accepted es authentic but whose origin can be attributed to the transmitter or the recelver or to other third parties with the object of making it appear that one of them is attempting to subvert the system or to undermine the user's faith in the comunication system, or he may wish to alter genuine messages so as to have them rejected as unauthentic. Finally, the receiver may have all of these objectives plus others. He may wish to elther create forgerles or to alter genuine messages and have them appear to be authentic, he may wish to reject authentic (unaltered) messages as unauthentic or to alter anthentic messages so that they can be rejected. He may also wish to accept an unauthentic message as authentic either with the objective of not letting the transmitter or third parties know that he has detected that the message is unauthentic, or of accepting it with the intention of at sane later date rejecting it as unauthentic or perhaps of forcing the trancmitter to accept responsibility for a message which he (the receiver) knows to be unauthentic.

In all of the cases just described what is meant by a party achieving their objectives is not simply that they be able to carry out an act, but rather that when the evidence on which the act was based is presented to still another party acting as fudge or arbiter that the party who acted contrary to the intent of the secure communications system will be held blameless, 1.e., that the judgement will be that they acted in logical accord with the information available or that there is insufficient evidence to prove that they acted to subvert the communication.

If we now add considerations of secrecy to those of security, the problem becomes even more involved. It is usually assumed that the transmitter and receiver desire to communicate in secrecy, but that some third party wishes to be privy to their private colmunication. In fuct ellher the transmitter or the recelver may wish to have the secrecy violated while the third party may wish to preserve it. For example, a nation acting as an intermediary in a very delicate diplomatic negotiation between two otber nations might wish to keep secret the details of the negotiation because of the possible consequences to them if their role became known while one or the other of the communicants might wish to have them revealed under circumbtances were one of the other parties could be blamed for the breach of security. Thus, it may be that either the transmitter or the receiver wishes to Flolate the secrecy of the communications (while not appearing to do so), while third parties may have the more comon objective of penetrating the secret communications, elther for their own information or so that it can be made to appear that one of the transmitter or recelver (or perhaps still another third party) compranised the secrecy of the communications.

Finally, in adition to situations wherein only one of secrecy or security is of concern there are also situations in which they are of joint concern. Usually, both functions are ostensibly desired by the transmitter and the recelver, however, as the present author has pointed out 5,6 in studies of systems to verify internetional compliance with treaties, there are instances in which euthentication is required but in which secrecy is not only not needed but in fact is prohibited that give rise to some of the most involved secure communtcations problems yet encountered. In each of these enviroments, the various participants may have any mix of the deceitful objectives already described or equally importantly may wish to convince third parties that one of the others has worked one or more of the deceits.

Every objective described for the subversion of the intent of secure communications has arisen in real-world diplomatic and milltary communications situations - and most bave plausible analogs in commercial and private sector communications. The title to this paper was chosen because in the worst case, deceitfui objectives can be attributed to every participent to the communication process. Since the objective of any secure communications system is to thwart deceit, this paper will investigate what can be done in some specific instances.

\section{The ABCs of Authentication}

We ossume that the reader is familiar with the essentials of contemporary cryptography, 1.e., that he knows about the Federel Data Encryption Standard (DES) $7,8,9$ as representative of symetric (one-key) systems and is also generally acquainted with the principles of asymetric (read public-key or tworey) cryptography.1,4,5,10,11 While not essential to the understanding of this paper, the reader would be much aided by having read one or more of the several available papers that compare and contrast symmetrlc and asymetric cryptography $5,17,12$ It is Infeasible to re-cover these points in this paper so that much will be taken for granted that requires careful discussion at some place.

We shail use a very simple communications problem as a vehicle to discuss authentication. A commander has a mumber of subordinates whose function It is to execute a serious and irreversible act, ouch as firing missiles, on recelpt of orders to do so. If both parties trust each other, then the system at its simplest need only provide authentication to the subordinate that a fire order came fram his 
superior. This could be sccomplished by incluaing a preagreed upon, but secret from all other parties, message $(s 1 \mathrm{gn})$ in the fire order. If the concern is only with accidental misunderstanding or with an outsider attempting to impersonate the superior commander, such a naive safeguard might suffice. It could not protect against a third party who intercepted the commander's order to learn the sign and then included it in a false order to the subordinate in a "postal chess" ploy.

Authentication in its simplest terms is the determination that a received message is in the subspace of acceptable messages $M$ of the space $m$ of all possible messages. In the scheme just described, $M$ is the collection of messages that include the sign and $m / M$ are all of the messages that don't. If $m$ is encrypted to a cipher $c$ with a secure cryptosystem, then an opponent (third party) substituting some other cipher $c$ ' would have a probability of no better than $|\mathrm{M}| /|\mathrm{m}|$ of having $c$ ' be accepted os authentic.

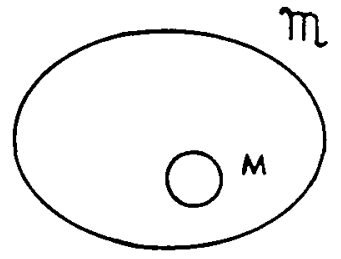

Continuing the simple set disgrams illustratIng authentication, there are three spaces (or sets) in a cryptosystem: the messages or plaintext, $m$, the reys, $x$, and the ciphers, $c$.
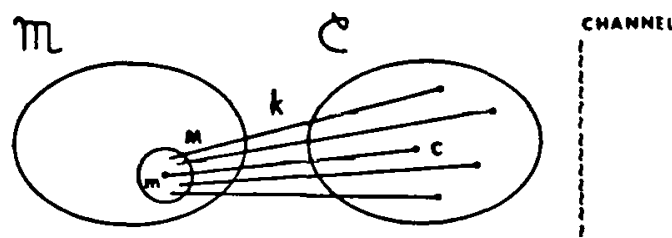

The above flgure illustrates the third party's dilemma since even if he knows $M, C$ and $M$, and even $m$ and $c$, he can do no better than guess at an acceptable cipher $c$ ' if the cryptosystem securely conceals the key $k$. Therefore, we shall restrict attention to thwarting the deceitful objectives of the commander and his subordinate in the rest of this discussion.

If the superior commander requires verification of receipt of the order, another prearranged message (countersign) fram the subordinate would have to be returned. In Pact, many military command and control situations are as simple as this. Either party can claim to have received the prearranged 1dentifiers when in fact they didn't (which in this case is equivalent to their being able to generate undetectable forgeries, 1.e., to select any other $m \& M$ ), so that this system can only detect unauthentic messages introduced by third parties, but cannot protect against deceit by either communicant. Because authentication, 1.e., the determination that a message is in $M$, is invariably dependent on recognizing the presence of an already known or derivable (to the anthorized users) redundancy in the message, the receiver can only be prevented from making for- geries by denying him the ablifty to introduce the requisite redundant information into a fraudulent message. It is one of the main objectives of this paper to explore fully how this is accomplished using elther symetric or asymetric encryption techniques and to determine the differences as sherply as possible.

Authentication against deceit by insiders can be achieved using a symetric encryption system such as the DES in a simple variation of the camon way such systems are used to provide password file security in computer log-in systems. The problem with an unprotected, 1.e., raw, user password file Is that anyone having or gaining access to the file could impersonate any user by submitting the user's Identifying password. The solution, proposed by Needham, 18 is to file not the user's password but a function of the user's password where the function - which must be exposed in the computer, of course - is noninvertible or "one-wey." Almost from the beginning it was recognized that any secure cryptosystem could be used as a one-way function to provide the desired password file protection. In a symmetric system, the cipher (message) can be found given the key and message (cipher) while the key cannot be recovered from a knowledge of the cipher and message (known pleintext attack), hence $a$ one-way function exists between the key and the message-cipher pair. In password file protection the user's password is therefore the key and the message is some fixed test phrase that is encrypted with each user's key into the cipher stored in the file. Anyone having access to the encryp-

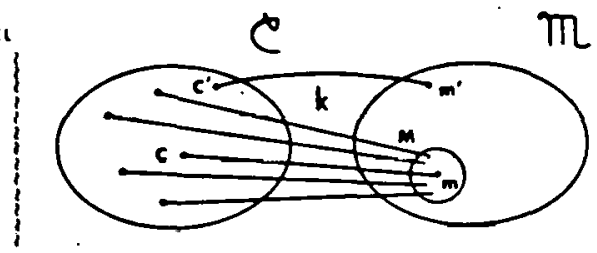

tion algorithe, the test phrase and the cipher file would still be unable to impersonate a user, while a user can reliably authenticate himself by presenting the rey that encrypts the test phrase to a cipher that matches his stored cipher. Lamport 4 was apparently the first to note that this seme technique could be used to provide a one-time authentication of a transmitter to a receiver. The reason that it can only be used once for this purpose but repeatedly for access to the computer is that while the computer is assumed to not record the key nor to have deceitful objectives, it is decelt by the receiver that the authentication system is attempting to prevent. The receiver doesn't know the key until the transmitter reveals it - and thereafter he does. One way to use this idea in the present exemple would be for the cammander to select a message and a key with which be computes a test clpher. He then gives the test cipher and message to his subordinate but keeps the key secret to himself. When the superior wishes to order the missiles fired he sends his key as an authenticator. The subordinate decrypts the test cipher and matches the messages to verify the authenticity of the order. He can later prove that hé recelved the order from his superior since he can exhibit the key that 
he could only have obtained by elther receiving it from his superlor commander as arranged or by breakIng the DES to recover the key from the known plaintext and the test cipher. The return acknowledgment could be handled in a similar fashion. What has been described is a mapping of $c$ into $m$ by a fixed point $\mathrm{k} \in \mathrm{x}$. But by hypothesis $\mathrm{k}$ cannot be recovered from a knowleage of $m$ and $c$ so that such a scheme 1s cryptosecure.

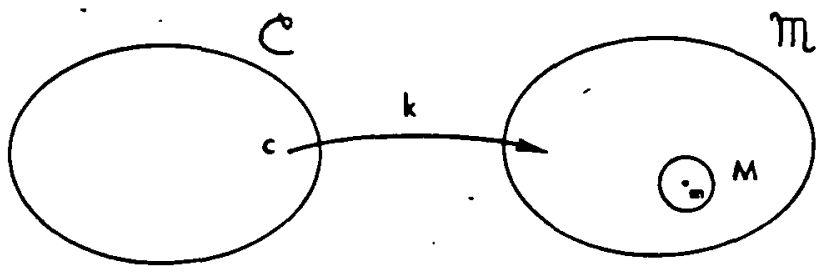

In this example only the decryption of $c$ by the specific key $k \in K$ selected by the superior commander would be accepted as authentic and the subspace of acceptable messages consists of only the single element in. One could equally well 8 ay in this case that the mapping is of $K$ into $m$ by the flxed point $c \in C$. When only a single message-cipher-key triple is involved, these are indeed functionally equivalent. As we shall see later there is a $\mathrm{sig}-$ nificant difference when $|M|>1$.

Even though authentication has been provided, there are still possibilities for deceit. Unless the original exchange of test cipher and message is witnessed and securely stored by a third party, elther party could later generate a key and cipher pair matching a fraudulent message from $M$ and assert that these are the true pair and that the key was recelved from the other party as authentication for a comunication, or conversely, disavow a true pair as fraudulent. A third party or arbiter would not be able to resolve the dispute.

Much more serious though in the scheme just described is the limitation that it is only possible to cormunicate an authenticated signal m to execute some already agreed-to plan, in other words the key $k$ can only be revealed (or used) once. If two or more messages $m$, were known to be authent1cated by the same $\mathrm{x}$ the receiver could substitute any one of these messages in the same way that he could choose among all of the membcrs of $M$ earlier, since the authenticator only proves that the superior commander has sent one of the messages - not that he has sent a particular message. A one-time authenticator is in a sense message independent much like the commercial codes of a half century ago, so that the wessage whlch is to be outhenticated must be agreed to in advance (and witnessed, etc.). This was a common problem with conmercial. codes such es $\mathrm{ACME}^{*}$ in which only messages that were in the codebook could be efficiently transmitted.

* The ACME codebook consisted of 100,000 fiveletter code groups out of the $26^{5}=11,881,376$ possible groups, i.e., $|m|=11,881,376,|M|=100,000$ so that the probability that a randomly chosen five-letter group is in the codebook is $\leq|\mathrm{M}| /|\mathrm{m}|$ $<0.85 \%$. To designate a point in $m$ requires the comunication of $\approx 23.5$ bits while a message only
By prearranging a large number of battle plans and ciphers (1.e., by enlarging the codebook), an execute signal could be authenticated for any part1cular plan by revealing the appropriate key. It is in this more general setting that the subleties of authentication are revealed. As has already been remarked, one cannot use the same $\mathrm{B}$ to authenticate all of the messages. Schematically, the analog to the single message-cipher case for multiple messages would be

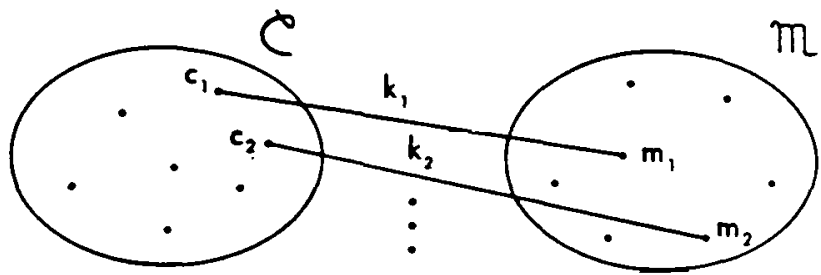

where the $x_{1}$ could elther be chosen to be all different or randomly chosen from $X$ with negligible effect on the authentication security. The scatter of the points of $M$ in $\mathrm{m}$ and of their ciphers in $\mathrm{C}$ in the figure is intended to suggest that neither is capable of a succinct (functional) description but that a tabular description of both subsets would be needed. Obviously, one or the other of these two subsets could be chosen to have a simple description and the other (computed using the $x_{i}$ ) would be devold of structure,"i.e., would have to be tabulated if the $k_{1}$ are to be concealed. As a consequence of $x$ being the key space for a secure cryptosystem it doesn't matter whether the points in the one set are drawn randomly or chosen to be near each other using some metric or in fact irrespective of any relationship that they may have in the containing set, the images are randamy scattered in the other set. What is more, since the cryptosystem is immune to a known plaintext attack, there is no computationally feasible way for a user who is given the cryptosystem to 1mpose order or structure on the images since this would be tantamount to solving for keys given plaintext information and clpher - which by hypothesis cannot be done. For example,

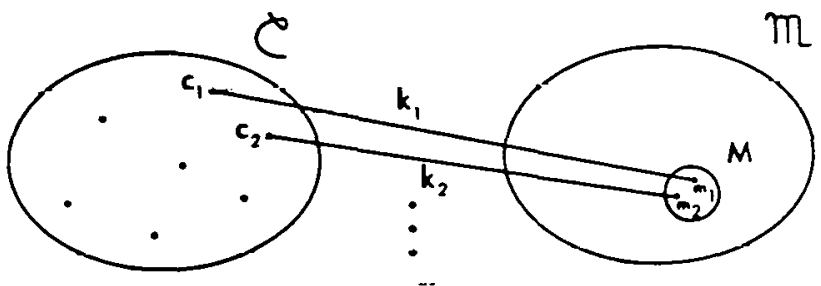

contains $\approx 16.6$ bits of information. The remaining $\approx 6.9$ bits of redundant information would in this analogy be the authenticator. The entry in the codebook and the code are unrelated: the curious and certainly infrequently needed messages

OYDEU "Charterers paying towage to Humbolat Bay and out to sea"

FIFUB "Must have a good red label"

can be transmitted efficiently using the indicated codes while "Cambodia has been invaded by Vietnam" would require almost a letter-by-letter encoding since neither country's nome is in the codebook. 
or the converse. The restriction that the party wishing to subvert communications not be allowed to design the cryptosystem is essential. Otherwise it is possible for him to design a cryptosystem on a rey space $X$ which maps $M$ into $C$ and then to extend the functions to map in into $c$ while preserving the kernel mapping of $M$ into $C$. In fact, it is possible to construct large numbers of cryptosystems that share kernels so that $m_{i} \varepsilon M$ is encrypted into $c_{1} \in C$ for all $k \in K$, but in which m $\in M M$ is mapped randomly into $C / C$. Such a "separable variables" scheme would Introduce a dangerous "crypto weakness if the receiver were given the ordered $M$ and $C$. The way to avold this problem is similar to the classical rule for dividing a cake fairly - let one party design (or choose) the general system and the other use it. In the case of DES so far as anyone knows, in spite of suspicions to the contrary, 19,20 It is Impossible for anyone to solve for the keys that would permit both message and cipher subsets to be sinply described (ordered).

As described above, either the tabulation of the pairs $\left\{m_{i}, c_{i}\right\}$, or the description of $M$ (indexed by 1) and a tabulation of $\left\{c_{j}\right\}$ or conversely the description of the ciphers $C^{1}$ (indexed by 1 ) and a tabulation of $\left\{m_{i}\right\}$ would be given to both the intended recelver and to the arbiter in advance of comminication. The transmitter would outhenticate a message $m_{1}$ by revealing key $k_{1}$.

Clearly, only the tabular listing or the functional description of $C$ has to be revealed in advance in order to permit authentication. The transmitter would then reveal the missing pair of elements, $m_{1}$ and $k_{1}$, to authenticate a transmission. Let $\left\{c_{i}\right\}=C$ be the published list, then the recelver has only to find some key-message pair whose cipher is in the table in order to be able to utter an undetectable forgery. But for any rey $k^{*}$, there is some cipher $c_{i}$ which is the encryption of $m_{j}$. Therefore the chance that $c_{i}$ when decrypted by $\mathbf{~}^{*}$ will be in $M$ is $|M| /|M|$ - so that we have a variant of the "birthday problem" in searching for arbitrary key - cipher pairs whose message is in $M$ with perhaps unacceptable authentication security as $|M|$ becomes large. 21 If $M_{\text {_alone were given to the }}$ receiver, irrespective of whether it was an unordered latular listing or a functional description, he could choose any $m \in M$ and encrypt it with an arbitrary key is to obtain a clpher $c_{j}$. He could then claim that this pair had been sent to him by the transmitter as authentication for m.

The conclusion is that the receiver (and the arbiter) must be given $C$ and may also be Given $M$ in order that they may authenticate messages as having come from the transmitter. The probability of the receiver being able to falsely attribute a message to the transmitter in the two cases is different, but potentially acceptable in eitber case. For this reason, in all variants of this scheme, the receiver and the arbiter must be content with a codebook to permit them to decide if a message is to be accepted as authentic or not.

The crucial difference between symetric and asymetric systems in simple message authentication is that it is possible to avoid the need to distri- bute the random entry codebook in advance! In the direct analog to the authentication system just described using symetric cryptosystems the existence of the two reys $d$ and $e$ that act as functional inverses to each other in an asymetric system makes it possible for the transmitter to compute a cipher $c$, using $e$, that will decrypt into $m \in M$ using $d$. Therefore, the recelver and arbiter could be given $\alpha$ and $a$ succinct (functional) description of $M$; for example all acceptable messages will end in a time, date and message muber. Since $e$ is concealed from a znowledge of a by a computationally infeastble task, the receiver even though he knows $M$ has no better chence of constructing a cipher $c^{\prime}$ which will decrypt into an m' $\in M$ than does a third party who mist guess at ciphers. The transmitter on the other hand authenticates messages simply by being in possession of a cipher that he can reveal that decrypts into an acceptable message.

It is also possible in an asymetric system to give the receiver and the arbiter the decryption key in advance and then to authenticate a message by presenting a message-cipher pair in which the cipher will decrypt using the recelver's vey to the message. This is in fact the way that the authent1cated access system designed by Sandia for the zero power Plutonium reactor at Idaho Falls works. The esymetric decryption rey is stored in the access control equipment (computer). The message includes the Individual's hand measurements (left and right) and same other physical characteristics which are originally encrypted at a secure location, with positive identification of the individual, etc., and recorded on a magnetic ID strip on the back of his badge. When he later wishes to be granted access to the plant he places his hand(s) in the scanner which creates the "message" and his badge in the magnetic reader. The cipher is decrypted and the resulting messages matched. A perfect match is not required since the message is so redundant and difficult of forgery. The important point in this version is that no codebook exchange occurs in advance of communication - only the authenticated insertion of the decryption key.

There are some hair-splitting points about just what is key, and how many bits of information are actually comunicated in the various schemes - but the essential difference is what has just been pointed out; that the exchange of the random entry codebook in the symmetric schemes has been replaced by either the exchange of a succinct structural description of the messages (or of the ciphers) and a functional description of the noninvertible randomizing decryption function or of only the decryption function. It is almost as if the codebook virtually existed in the asymetric system, but that the receiver can only look up those entries required to autbenticate messages received from the transmitter!

When put in such simple terms, the significance of asymmetric cryptography is hard to appreciate. It wasn't for this reason that we said earlier that there was less to it than meets the eye, for indeed the capability to solve some otherwise imposbible problems lies mainly in the abjilty of twokey systems to authenticate commications where 
the exchange of codebooks would be impractical.

\section{How to Authent 1 cate a Key Directory}

In the introduction it was pointed out that whereas the keys must be exchanged in secret in a symetric system that they need only be authenticated in an asymetric system. Even this statement true though it is - is more complex than it seems. To see why this is 80 , consider the simple problem of establishing what has been called a CKA (centralized key authority) in a symetric system which is the functional counterpart to the publisher of the directory (the PKD) in a public-key system. The CKA must authenticate the reys submitted for inclusion in the file in the sense that be must be able to vouch for the 1dentity of the party submitting the key and of the party requesting the issuance of a key. There are instances in which this 1sn't the case. For example, a pawnbroker doesn't care who presents the ticket to reclaim a pledge, but the consequence is that there is a marret in pawn tickets. The more commonly cited counterexample is that of a remote data terminal that pretends to be one of a bank's autcomatic tellers. In this case, the argument is that since the data terminal has no funds to disburse it is immaterial whether it can falacly solicit and recelve an order from the banks central computer to disburse cash. There are other deceitful objectives which it could accomplish though, such as the famous case in which a customer substituted for the bank's counter deposit slips his own deposit slips bearing his account muber on the magnetic ID strip instead of the bank's open account number. The result was all the other customer's deposits were credited to his account.

If $A$ wishes to communicate with $B$ in a symmetric CKA system he does so by sending an authenticated message to the CKA that may either include the session key $k_{A B}$ to be relayed to $B$ by the CKA with appropriate authentication, etc., or else $A$ requests the CKA to choose a key and forward it to $A$ and to $B$ along with the request that communications be established. A cen only be certain that he is actually in communication with $B$ if he can rely not only on the integrity and security of the CKA but also on the quality of the data in the CKA' Piles. His confidence in the authenticity of a communication from the CKA is derived from his confidence that only the CKA's privileged data can make such a communication possible. A great deal has been written about protocols $14,15,16$ to achieve these purposes which we shall not re-cover here except to remark that the CKA's function is possible if and only if the conditions described in the preceding section for one of the authentication schemes are satisfied.

The system described above does not protect the subscribers Pron deceit by the CKA. Protocols involving redundant but parallel CKAs have been devised for this purpose, 16 but this only says that there is security in mubers, not that the need for authentication has in anywise been replaced or reduced.
In an asymotric CKA-type system the clatm (and bejlef) was that each subscriber, 1 , could generate a pair of keys, $d_{i}$ and $e_{1}$, and submit the encryption key, $e_{1}$, without the need for authentication, to a FKD who would publish a public rey directory, while the subscriber would reep his decryption key secret. There are several flaws in this. First, the PKD can never do more than vouch for the directory being an accurate reproduction of the data submitted to him or collected by him or his agents. The PKD cannot in the preparation of the directory detect an "active wiretapper" who intercepts the camunication of $k_{B}$ by $B$ and substitutes $\mathrm{k}$. In this respect the $\mathrm{PKD}$ is like the pubIisher of a city directory who by proofreading, etc., can only verify that the directory is an accurate reproduction of the input information. In both cases the publishers can only be certain of the accuracy of the information in the directory after each user has verified his own entry. Error detection in fact comes mainly from third parties who discover errors in using the directory, but this is not an analog to the PKA since no subscriber can verify another party's entry in this case. While it is true that the publisher could be certaln the directory was correct if each subscriber verified bis entry, it is not sufficient that he receive no complaints after the directory 1s distributed - even though it is required that every subscriber check his entry within some time perlod. One reason is that the active wiretapper who intercepted $B^{\prime} s$ comonication of $\mathrm{K}_{\mathrm{B}}$ and substituted $k_{B}^{*}$ could have created a fraudulent directory (for B's exclusive deception) which has B's correct key of, $\mathbb{k}_{B}$, in $1 t$. The active wiretapper could also simply wait until the directories are published and intercept any that he wishes (to $B$ say) and modify any key(s) other than that of the recipient. In either of these circumstances, B would not detect the deception. The result is 1denticul in both cases except that in the first he enlists the FKD as an unwitting accamplice in his dietribution of forged keys. All that the PKD cen conclude from the lack of response by the subscribers is that none of them detected errors in their entries in the drectory - which says very little about the accuracy of the directories in circulation. It is also possible that the opponent is in a position to prevent $B$ from notifying the PKD that his zey is incorrect - even if he did recelve a directory containing $\mathrm{k}_{\mathrm{B}}^{*}$ instead of $\mathrm{k}_{\mathrm{B}}$.

What is needed of course is a means of authentication for directories - which at first seems contradictory, since the need (from the PKD's point of view) for authentication (of directories) is due to the imposibility of autbentication of airectory entries. The reason that a solution may be possi-. is the difference in what is being authenticated in the two instances. If, for example, the keys are sixty-four bits in length and the directory contains one-million entries, then in the forward (subscriber to $\mathrm{PKD}$ ) direction one-million messages would have to be authenticated at whatever level of security is required. In the reverse direction, only one message, consisting of sixty-four million bits it is true, would need to be authenticated to the same level of security. Recalling the earlier discussion of authentication prificiples nne r.an 
see that while a substantial fraction of the total communication would be redundant in the forward direction only a vanishingly small fraction of the message need be redundant in authenticating the directory.

An aside illustrates the point of the preceding paragraph. The first authentication system on which the author worked for an ARPA application had to authenticate a $10^{8}$ bit data block with $\approx 100$ bits of redundancy. Side conditions made it impossible to encrypt the entire $10^{8}+10^{2}$ bits, so the solution was to use a secret hashing function (part of the key) and then to superencrypt the resulting $10^{2}$ bits so as to further conceal the hashing function. This is almost an exact analog to what is needed to authenticate a directory.

Assume, for the moment that an asymetric hashing/encryption function can be defined with which anyone possessing the encryption key could calculate a 64-bit authenticator for the entire dircctory. The probability of any change then going undetected would be $2^{-64}$ and the probability would be the same for anyone finding the authenticator matching an altered directory. It is then paradoxically possible to authenticate the directory - while the entries could not be authenticated to the PXD, using an 1dee first suggested by Merkle.17 If the PKD distributes the key and authentication cipher for the directory in sufficlently many ways, even though each channel is itself suspect, the cumlative effect is an authenticated transfer of a small amount of information. If radio, TV, major magazines and newspapers, direct mail, computer networks, etc., all carry the 8 ame message - rey plus authenticator - it becomes Improbable that even the most able and determined opponent could control all of these channels, with the result that the authentication channel provldes one reliable communication. The price for this reliability is a very low channel capacity. A few hundred bits might be disseminated so redundantly a few million would be inconcelvable. Now, subject to same constraints on the hashing/encryption function, each user can determine whether he has received a true copy of the directory published by the FKD. If each subscriber also checks his own entry (key) in the directory - lack of complaints will prove to the PKD not nnly that unaltered directories reached all "subscribers" but in addition that the directory is correct essuming of course that the subscriber responses reach the PKD. "Subscribers" is intended to indicate that the party who submitted the rey to the FKD also makes the verification. If an antbeuticated coliection system exists for the $\mathrm{FWD}$ to collect reys, the same system could be used to collect authenticated notices of errors. If no such authentisater colleotion system extsts, there will presumably be an enormous difference in the channel capacity required to feed back to the PKI errors in the directory us opposed to what would be needed to collect the keys initially so that it may be possible to solve the one problem but not the other. In any event, the PKD's ability to certify the directory is totally dependent on the subscriber's notices of errors reaching him. It is still possible for $C$ to impersonate $B$ if $B$ is not a subscriber and hence does not have a legitimate entry in the directory to be verifled. As in the symetric cese this decelt can only be prevented by requiring that the association of keys and subscribers be authenticated, which can be accomplished if an althenticated collection system exists but not by the low-capacity redundant channel postulated for directory autbentication.

A subscriber, A, may not1fy the FKD that he has detected elther of two kinds of error. A's entry may be correct and the directory not authentic which says that his copy of the directory is not a true replica of what the FKD published, or A's entry can be in error and the directory either be authentic or not authentic. In the first case, if there are no complaints from other subscribers, the FKD concludes that only $A^{\prime} s$ entry is incorrect. In the second case, even if other subscribers complain, the problem cannot be sharply identified. All that the returns show is that a number of subscribers have directorles with erroneous entries one of which is their own.

Thus far, we have not even considered the problem of a deceitful PKD. Only the PKD is in a position to know whether the directory has been authenticated or not. He could disregard communications notifying him of errors - although be could not in the scheme just described (with sultable constraints on the hashing/encryption function) be any more successful at foisting a fraudulent directory on a subscriber then a third party. In fact, what is desired is a protocol in which the PKD need not be trusted at all - except to publish the directory and to respond to subscriber complaints. If he fails to perform this latter function the subscribers who authenticated their directories would assume them to be correct and would presumably proceed to use them. The subscribers whose entries are incorrect or whose directories didn't authenticate would not use the directory - but a ciphered message from one of the decelved subscribers to ane of the subscribers whose directories were unauthentic could be misdirected to and responded to by a third party. For example, consider a threesubscriber directory which should contain keys $\mathrm{k}_{A}$, $\mathrm{k}_{\mathrm{B}}$ and $\mathrm{k}_{\mathrm{C}}$, but actually contains $\mathrm{k}_{\mathrm{D}}, \mathrm{k}_{\mathrm{B}}$ and $\mathrm{k}_{\mathrm{C}}$ with which the PKD calculates the authenticator. would notify the PKD that his directory wasn't autheitle and also would tell him that his entry was incorrect if he received a copy of the directory published by the PKD or that his key was correct if he received a directory containing $k_{A}$ as it should. If the FFD failed to forward this information, $B$ and $C$ would accept the directory as authentic and correct. They could communicate in secrecy and with authentication with each other, but efther $B$ or $C$ would be decelved into communicating with D in the wistaken bellet that they were in communication with A.

In the system just described, all of the information available to the PKD is also available to subscribers and presumably third parties. The PKD is privileged in the sense that he publishes the directory, which function can be usurped by an active opponent, but he also disseminates the key and authenticating cipher which function cannot be usurped and expects to receive the subscriber res- 
ponses reporting errors in the directory. As we have seen this makes it possible for a deceitful FKD to indetectably deceive some users as to the accuracy of the directory. If all subscriber responses reach the $F K D$ he can deduce the subset of subscribers who mutually share good keys, 1.e., a good subdirectory to the larger directory. It is difficult to see how this infarmation could be utilized if very many reports of errors flow in from subscribers.

One of the necessary conditions on the hashing/encryption function is that the order of the reys mist have an effect on the output clpher, otherwise an opponent could interchange his rey and $A$ 's zeys in any directory other than the one sent to $A$ and have the change go undetected. He would then be able to impersonate A. Most importantly the hashing/encryption function must not be invertIble to prevent an opponent from constructing a codebook with the same authenticator as was compiled by the PKD. Obviously, the inverse function Is many valued - but it is conceivable that all but one key could be fixed and the determinate problem of inverting the cipher to find the unique missing key solved. In fact, it was a version of this separation-of-variables approach that led to the need for the concealment of the hashing function in the ARPA system described earlier. Unfortunately, this approach is not applicable in the present situation since the PKD cannot be trusted and there is no physicaliy secure piece of hardware that functions in his stead. The only means of checking on a deceitful PKD is by having several operate in parallel, exactly as was done in the symetric CXA protocol. Also, fust as the only means for the CKA to vouch for the accuracy and integrity of the xeys in his file was for their submission to have been authenticated, the same requirements exist for the PXD function.

In summary we have described a system, with not too many belling-the-cat features, that allows the PKD to verify that he has published precisely what was submitted to him and that true replicas of the directory have reached the parties that submitted keys. He cannot be sure of the 1dentity of the party that submitted a rey in a subscriber's name unless the purported subscriber is in fact a suhscriber. Furthermore, a subscriber can verify that his own xey is correct in his copy of the directory and that he has a true copy of the directory published by the FXD. Assuming that subscriber responses can reach the $F K D$, then after confirmation of the correctness of the dinectory througb the redundant ccomunications channel, o subscriber can be confident that he has a true copy of the directory published by the PKD and that the PKD has correct.ly gublished the kcys submitted to him. On the negative side very many errors paralyze the cystem since there is no way to recover except by authenticated communications which are assumed infeesible. Consequently the system is very easy to sabotage - by any of the parties whose deceitful interests might be furthered by doing so. Also, a deceitful PKD can be made less likely by having several PKDs that publish duplicate directories in the hope that collusion to decelve is less likely than individual deceit. It appers that the authen- ticated channel capacity is in a sense inversely proportional to the ease of denving authentication to the final product - the directory.

\section{Summary}

The ralson diêtre for this paper was to clarify which secure comonications objectives could be realized using elther symetric or asymetric cryptosystems even though varlous of the parties to the commication are deceltrul. This is far too important an objective to allow the conclusions to be obscured by either the length of the paper or the labyrinthine discussion so we shall recap them here.

We pointed out at the beginning that an asymmetric cryptosystem either makes it possible for a transmitter whose key has been compromised to still communicate with secrecy to a recelver whose key has been protected, or else for a recelver to authenticate a message so long as the transmitter's rey has been kept secret. These are very important differences from a symetric cryptosystem in which both the transmitter and receiver keys must be kept secret if any secure comonications are to be possible. However, in the design of systems to cope with deceitful objectives by the transmitter, receiver or of third parties, it is a surprising finding that the same needs for authenticated exchanges and for trustworthy centers exist irrespective of whether symetric or asymetric encryption techniques are used! There are significant differences in the amount of information which the communicants must exchenge in advance in order to authenticate messages and in the requirement that the exchange be carried out in secret in the symmetric case while it can be done in public exposure in the other. But the essential point is thet twokey cryptosystems can only achieve authentication functions that can also be achieved by one-key systems - with the important distinction that in sowe instances the asffering requirements for the operation of the two makes the two-key system practical while the one-key system, though possible in principle, would be infeasible in practice.

\section{References}

1. R. Rivest, A. Shamir and L. Adleman, "A method for obtaining digital signatures and publickey cryptosystems," Commun. ACM 21, 2 (Feb. 1978), 120-126.

2. S. M. Lipton and S. M. Matyas, "Making the digital signature legal - and safeguarded," Data Cacmun. 7, 2 (Feb. 1978), 41-52.

3. M. O. Rabin, "Digitalized signatures," Foundetions of Secure Computation, $R$. Iiption and $R$. DeMillo, Eds., Academic Press, Néw York (1978), 155-166.

4. W. Diffle and M. E. Hellman, "New directions in cryptography," IEEE Trans. Inform. Theory IT-22, 6 (Nov. 1976$), 644-654$. 
5. G. J. Simmons, "Symetric and asymetric encryption," Computing surveys 11, 4 (Dec. 1979), $305-330$.

6. G. J. Simmons, Message authentication without secrecy: a secure communications problem unfquely solvable by asymetric encryption techniques," EASCON'79 Record, Arlington, VA (0ct. 1979), 661-662.

7. R. W. Roberts, "Encryption algoritlm for computer date encryption," (NBS) Fed. Reg. 40, 52 (March 17, 1975), 12134-12139.

8. R. Morris, I. J. A. Sloane and A. D. Wyner, "Assessment of the National Bureau of Standards proposed federal data encryption standard," Cryptologis I (July 1977), 281-291.

9. M. E. Hellman, R. Merkle, R. Schroeppel, L. Weshington, W. Diffie, S. Pohlig and P. Schweitzer, "Results of an initial attempt to Cryptanalyze the NBS data encryption standard," Electrical Engineering Dep., Stanford Univ., Stanford, CA, SEL 76-042, available from NTIS (Sept. 9, 1976).

10. R. C. Merkle and M. E. Hellman, "Hiding information and signatures in trapdoor knapsacks," IEEE Trans. Inform. Theory IT-24, 5 (Sept. 1978), 525-530.

11. W. Diffie and M. E. Hellman, "Privacy and authentication: an introduction to cryptography," Proc. of the IEEE 67, 3 (March 1979), $397-427$.

12. A. Lempel, "Cryptology in transition: a survey," Comput. Surv. 11, 4 (Dec. 1979), 285-304.

13. G. J. Popek and C. S. Kline, "Encryption and secure computer networks," Computine Surveys 11,4 (Dec. 1979), 331-356.

14. F. Horst, W. A. Notz, and J. L. Smith, "Some cryptographic techniques for machine to machine data communications," Proc. IEEE 63 (Nov. 1975), 1545-1554.

15. S. M. Matyes and C. H. Meyer, "Generation, distribution, and 1nsta.l lation of cryptegiuplic keys," IRM Syst. J. 17, 2 (1978), 126-137.

16. R. M. Needham and M. D. Schroeder, "Using encryption for authentication in large networks of computers," Commun. ACM 21, 12 (Dec. 1978), 993-999.

17. R. C. Merkle, "A certified digital signature," to appear Commun. ACM.

18. M. V. W1 1 kes, Time-sharing camputer systems, American Elsevier, New York (1568).

19. M. E. Hellman, "DES will be totally insecure within ten years," IFEE Spectrum 16, 7 (July 1979), 32-39.
20. D. Branstad, "Hellman's data does not support his conclusion," IFEEE Spectrum 16, 7 (July 1979), 41.

21. G. Yuval, "How to swindle Rebin," Cryptologia 3, 3 (July 1979), 187-189. 